\title{
AUTOMATIC ANATOMICAL LABELING OF ABDOMINAL ARTERIES FOR SMALL BOWEL EVALUATION ON 3D CT SCANS
}

\author{
Weidong Zhang, Jiamin Liu, Jianhua Yao, Ronald M. Summers \\ Imaging Biomarkers and Computer-Aided Diagnosis Lab, Radiology and Imaging Sciences, \\ National Institutes of Health Clinical Center, USA
}

\begin{abstract}
While segmentation of the small bowel on CT scans is helpful for diagnosis and surgery planning, it is challenging to reproducibly identify specific locations within the small bowel. Assigning anatomical names to the individual mesenteric arteries supplying the small bowel may enable more precise lesion localization. It also enables registration of the small bowel from CT scans before and after surgery. In this study, an automated anatomical labeling method was introduced for the mesenteric arterial vasculature. After segmenting the mesenteric vascular tree, features including blood vessel size, direction, relative position and branching pattern were calculated. Known anatomic names were assigned to the major abdominal arteries, and names encoding order and direction were assigned to the arteries arising from the superior mesenteric artery. Five CT scans were analyzed and experimental results showed $73 \%$ recall and $79 \%$ precision rate. To our knowledge, this is the first reported method to label the smaller branches of the mesenteric vasculature and will have applications for diagnosis and treatment of small bowel disease.
\end{abstract}

Index Terms - anatomical labeling, small bowel evaluation, image segmentation

\section{INTRODUCTION}

Computed tomography (CT) is well established for the overall evaluation of the abdomen and pelvis. It is one of the primary methods for the diagnosis of small bowel pathology [1]. Compared to other techniques, such as small bowel follow-through, enteroclysis, endoscopic tests, CT is convenient and noninvasive. However, radiologists have to visually examine CT scans slice-by-slice to evaluate the small bowel and the interpretative procedure is time consuming and potentially error-prone. Automatic methods to interpret abdominal CT scans would be very desirable.

To evaluate the small bowel, segmentation is required but difficult, due to the low image contrast and high tortuosity of the small bowel and its close proximity to other abdominal organs on CT scans. Motivated by the fact that mesenteric vessels are much brighter than the small bowel on contrast-enhanced CT scans and have close anatomic relationships with one another, we proposed [2] a novel mesenteric vasculature-guided method for small bowel region segmentation. In this method, the mesenteric sheet (points/surface where mesenteric vessels supply the intestines) was used as the reference to localize and segment the small bowel. However, the small bowel cannot be differentiated from the colon because both of them adhere to the mesenteric vasculature as shown in Fig. 1 and have similar intensity on CT scans. This problem can be solved by assigning anatomical names to the artery branches. By referring to the artery names, it is easy to observe positional relationships between arteries and the small bowel. Anatomical labeling not only simplifies the segmentation of targeted organs, but also allows matching of structures across subjects. Based on segmented small bowel and mesenteric vasculature with assigned anatomical names, it is theoretically possible to match the vessel tree and localize the truncated piece of small bowel using the preoperative and postoperative $\mathrm{CT}$ data.

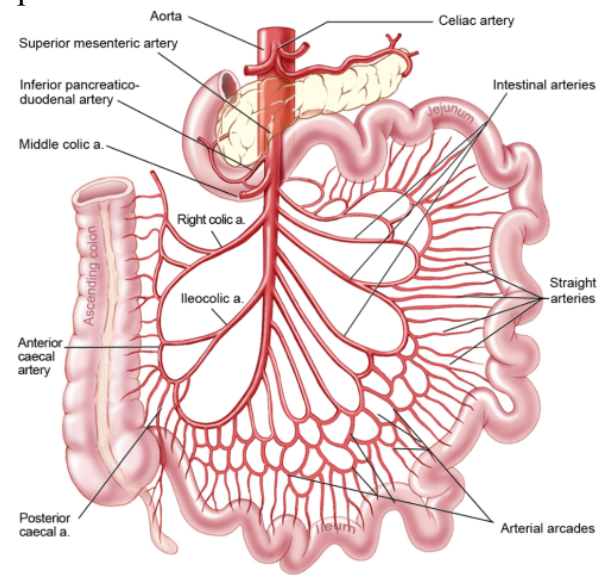

Figure 1. Mesenteric arterial vasculature of the intestinal system.

Anatomical labeling of the vasculature related to a target organ in abdominal region, such as the small bowel or colon, is very helpful. However, it is nontrivial since the topology of the vasculature is complex and varies from person to person. Furthermore, the segmented vessel trees 
differ between serial scans of a particular patient due to missing and spurious branches.

Several studies have investigated automatic anatomical labeling. Tschirren et al [3] labeled airway trees relying on branchpoint matching to a pre-labeled tree. Kitasaka et al [4] presented a method for automatic labeling of upper abdominal arteries using branching information. Mori et al $[5,6]$ presented a knowledge-based framework for anatomical labeling of bronchial branches and major abdominal arteries associated with the colon. No report was found for mesenteric artery anatomical labeling.

In this paper, we present an automatic anatomical labeling method for mesenteric and other major abdominal arteries on contrast-enhanced CT scans [7]. First, using spatial relationships of end-points and orientations of vessel segments, disconnected segments belonged to the same vessel branch are reconnected and then the complete vessel tree is generated. Second, major abdominal arteries are identified and labeled using size, direction, relative position and branching property. Third, first level mesenteric arteries arising from the superior mesenteric artery (SMA) are labeled in an incremental order. The method was evaluated on five abdominal CT scans and compared to visual assessment.

\section{INPUT DATA}

The data set consists of five high-resolution abdominal CT angiograms of patients suspected of having small bowel carcinoid tumors. Data acquisition and analysis were conducted under an Institutional Review Board (IRB) approved protocol. CT scans were acquired following oral administration of Volumen and intravenous administration of Isovue-300. The scanning parameters were section collimation $1.0 \mathrm{~mm}$, reconstruction interval $0.5 \mathrm{~mm}$, $512 \times 512$ matrix and in-plane pixel dimensions around 0.86 $\mathrm{mm}$.

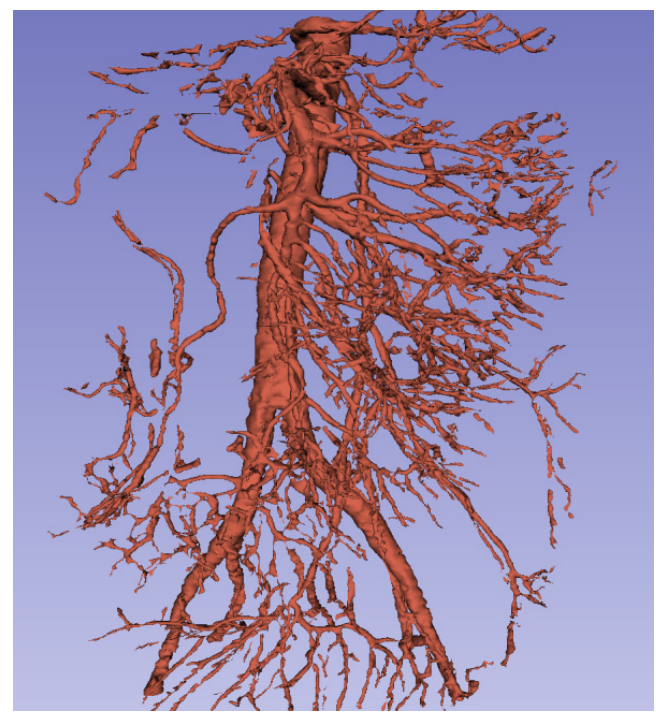

Figure 2. 3-D mesenteric vasculature segmentation.
For automatic anatomical labeling, the input is the mesenteric vasculature mask obtained using our previous methods [2, 7-10]. First, the internal abdomen region was extracted to reduce computation time and false positives, by identifying body contour, fat [8], spine [9], pelvic bones, and muscle [10]. Second, the mesenteric vasculature was segmented by combining vessel tracing and multi-scale vesselness enhancement on maximum intensity projection (MIP) images $[2,7]$, where non-vessel line-like structures and non-mesenteric vessels are removed. The segmented mesenteric vasculature is shown in Fig. 2.

\section{METHOD}

As discussed, there is a close anatomic relationship between the bowel and the mesenteric vasculature. The mesenteric artery branches follow an order such that the upper artery branches supply the duodenum, the middle branches supply the jejunum and the lower branches supplies the ileum and proximal colon. The ordered branching pattern can help differentiate the small bowel from the colon and segment the small bowel roughly into its three constituent parts. It is required to develop a system that can recognizes the vessel branches and labels anatomical name to each branch.

Our method automatically labels the major abdominal arteries and the arteries that arise from the SMA. The major arteries are the abdominal aorta (AO), celiac artery (CA), SMA, right renal artery (RRA), left renal artery (LRA), inferior mesenteric artery (IMA), right common iliac artery (RCIA), left common iliac artery (LCIA). The method also labels the first level artery branches arising from the SMA. Fig. 3 shows the structure of these target arteries.

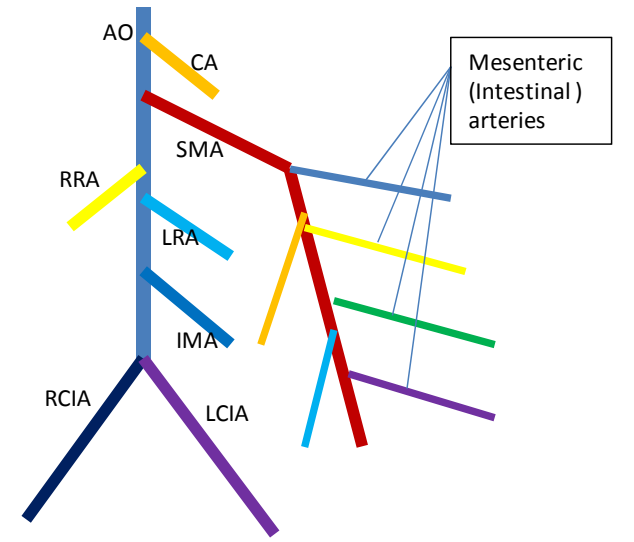

Figure 3. Schematic of abdominal and mesenteric arteries.

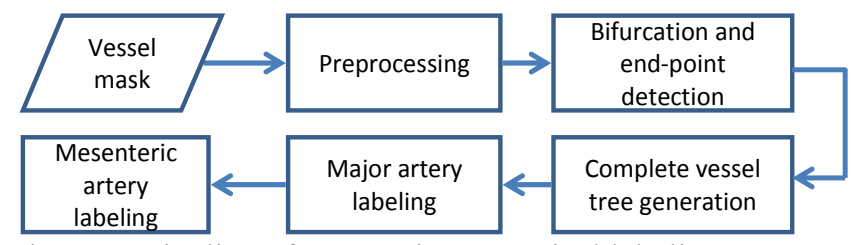

Figure 4. Pipeline of automatic anatomical labeling. 
Figure 4 displays the pipeline of our automatic anatomical labeling algorithm which can be divided into three steps: generating the vessel tree, labeling AO, CA, SMA, RRA, LRA, IMA, RCIA, and LCIA, and labeling first level mesenteric arteries.

\subsection{Preprocessing}

As shown in Fig. 2, many small vessels are detected and the resulting structure is very complex. To assist the anatomical labeling, small vessels and noise need to be removed. First, dilation and erosion morphological operations were performed to smooth the vessel mask. Then, Hessian-based filters with specified scales $(3-5 \mathrm{~mm})$ were used on vessel voxels (except the separated tracing results of AO, RCIA, LCIA and SMA as they should be kept in the final result), and small vessel voxels were removed by thresholding (bigger value for removing most of them and benefiting the final robustness) the corresponding vesselness values.

\subsection{Complete vessel tree generation}

To understand the branching pattern, it is necessary to detect the bifurcation points and endpoints of vessel segments (a bifurcation point can be seen as a special endpoint as it is an endpoint of two or more segments). We modified the connected component method [11] to detect these points-ofinterest. A hollow circle with fixed inner and outer radii (6-8 $\mathrm{mm}$ for AO, RCIA and LCIA, 3-4 mm for others) centered at a voxel was first placed. Then the number of connected components inside the hollow circle was counted. The points-of-interest can be easily detected since bifurcation points have more than two components, while endpoints of vessel segments have only one component.

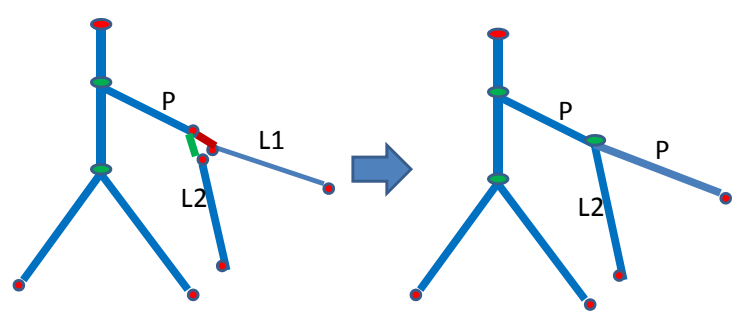

Figure 5. Illustration of reconnection procedure. Green points are bifurcation points, red points are endpoints.

As shown in Fig. 2, some of the detected vessel branches have discontinuities which should be reconnected. For each endpoint (except the root point of AO), taking the endpoint of vessel segment $\mathrm{P}$ (a 3D connected component represented by a matrix of $3 \mathrm{D}$ coordinates of voxel locations) shown in Fig. 5 as an example, we applied principal component analysis (PCA) to P, and took the first axis of PCA as the orientation of the vessel segment P. Nearby disconnected endpoints, which are not reconnected to others, such as L1 and L2 in Fig. 5, were searched and PCA was applied to their segments. As the orientation of L1 is similar to that of $\mathrm{P}, \mathrm{L} 1$ is reconnected to $\mathrm{P}$. We consider two vessel segments have similar orientation if the difference of their orientations is less than certain predefined value $\left(18^{\circ}\right)$. L2 is reconnected to $\mathrm{P}$ as a child-branch since they are not in the same orientation, but no other nearby segments can be found with which to reconnect L2. With the reconnection, corresponding endpoints were converted to bifurcation points, while the others remain as root and leaf nodes of the vessel tree.

\subsection{Major abdominal artery labeling}

With updated bifurcation points and endpoints, the vessel tree was built. Bifurcation points and endpoints are nodes, vessel segments between nodes are edges. Along the vessel tube, vessel diameters were calculated by taking the shortaxis length of local connected components.

Anatomic knowledge is applied to label the major arteries automatically. The algorithm first calculates vessel segment size, location and orientation, and then identifies specific segment using anatomic knowledge. The diameters of AO, RCIA and LCIA are much larger than those of other arteries and the AO passes though the center of the body and bifurcates into the RCIA and LCIA. Utilizing this knowledge [6], AO, RCIA and LCIA were identified and labeled. Using our mesenteric vasculature segmentation method [7], SMA can always be detected. The SMA was identified and labeled using the knowledge that the SMA has more child branches than other arteries arising from the AO. Then, CA and IMA were labeled utilizing knowledge that CA is located superior to the SMA while the IMA is located inferior to the RRA and LRA. Finally, RRA and LRA were labeled using their known lateral orientation.

\subsection{SMA-related artery labeling}

As our purpose is segmenting the small bowel accurately and comparing pre-operative and post-operative scans, what we need is to record the orders, directions and relative positions of arteries arising from the SMA. We labeled all branches from the same side in increment order, where PCA was applied to the local connected components near bifurcation points. The vessel having similar direction to the previously labeled SMA was labeled as SMA, and the other branches were labeled as SMA left or right child-branch according to the calculated direction.

\subsection{Performance evaluation method}

To assess the accuracy of our method, recall and precision were calculated:

$$
\begin{aligned}
& \text { Re call }=T P /(T P+F N) \\
& \text { Pr ecision }=T P /(T P+F P)
\end{aligned}
$$

where $T P$ is the number of branches correctly labeled, $F N$ is the number of branches that should be labeled but were not 
labeled, and $F P$ is the number of branches incorrectly labeled. Recall rate (i.e. sensitivity) measures the proportion of actual positives which are correctly identified as such. Precision is the proportion of positive test results that are true positives [6].

\section{EXPERIMENTAL RESULTS}

An anatomical labeling result is shown in Fig. 6, and the recall and precision rates are shown in Table 1 . The total number is the number of manually labeled branches, the detected number is the number of branches detected, and the labeled number is the number of labeled branches.

Errors for the major arteries usually happened on branches of RRA, LRA and IMA. Missed branches by detection method and errors in the reconnection procedure contribute most of the false negatives. Because of the performance limitation of the vessel detection method, some of the expected branches are missed. When bifurcation points are close to one another, errors will occur during the reconnection procedure. As shown in Fig.6, the second level branch marked "FN" attached to SMA-L4 should be one of the first level child-branches of SMA. False positives are mainly caused by vein branches. Unenhanced veins lie in close proximity to the enhances arteries. Nevertheless, some of vein branches were still detected and reconnected to the SMA, such as SMA-L3 shown in Fig.6.

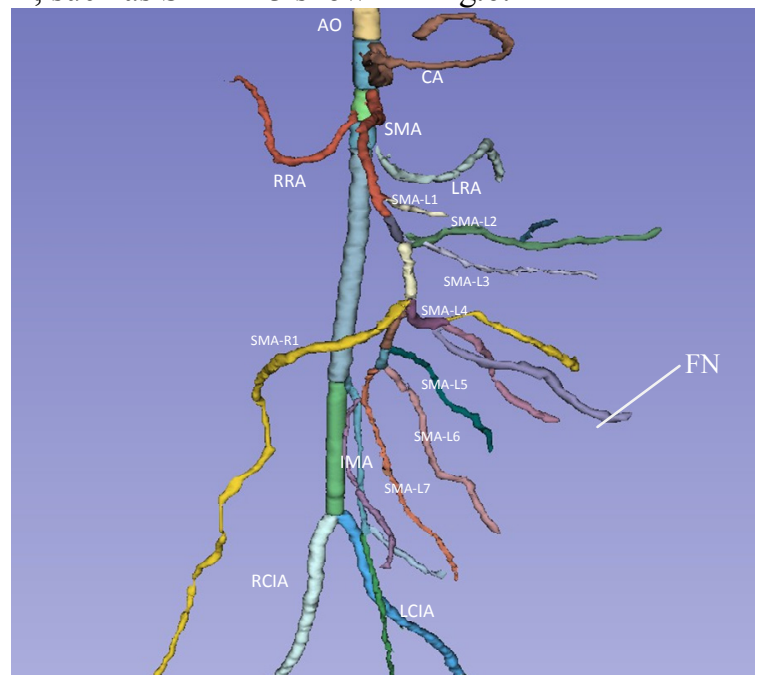

Figure 6. Anatomical labeling result for the example as shown in Fig. 2.

Table 1. Results of anatomical labeling.

\begin{tabular}{|c|c|c|c|c|}
\hline & $\begin{array}{c}\text { Total } \\
\text { branch }\end{array}$ & $\begin{array}{c}\text { Detected/labeled } \\
\text { branch }\end{array}$ & $\begin{array}{c}\text { Correct } \\
\text { labeled }\end{array}$ & $\begin{array}{c}\text { False } \\
\text { positives }\end{array}$ \\
\hline Case 1 & 16 & $17 / 15$ & 12 & 3 \\
\hline Case 2 & 17 & $17 / 16$ & 11 & 5 \\
\hline Case 3 & 16 & $15 / 15$ & 13 & 2 \\
\hline Case 4 & 16 & $18 / 16$ & 13 & 3 \\
\hline Case 5 & 17 & $18 / 14$ & 11 & 3 \\
\hline \multicolumn{3}{|c|}{ Recall rate $(\%)$} & \multicolumn{2}{c|}{ Precision rate $(\%)$} \\
\hline Total & \multicolumn{3}{|c|}{$73.4 \pm 8.3$} & \multicolumn{2}{c|}{$79.0 \pm 6.5$} \\
\hline
\end{tabular}

\section{CONCLUSIONS}

To help segment small bowel and match pre-operative and post-operative CT scans, we presented an anatomical labeling method for abdominal arteries including the first level arteries arising from the SMA. The method assigns a name to each branch of the complete vessel tree using size, direction, position, branching properties and order information. The method performed satisfactorily, with good precision and recall. The result was a number of named vessels sufficient to produce a coarse coordinate system along the length of the small bowel. This coordinate system will prove useful for lesion localization and for disambiguation of the small bowel path. Future work includes evaluating the method on a larger dataset and attaching anatomic names to arteries coming from the SMA instead of order names.

\section{ACKNOWLEDGMENT}

This research was supported by the intramural research programs of the National Institute of Health Clinical Center.

\section{REFERENCES}

[1] M. P. Federle, "CT of the small intestine: Enterography and angiography," Supplement to Applied Radiology, pp. 55-62, 2007.

[2] W. Zhang, J. Liu, J. Yao et al., "Mesenteric vasculature-guided small bowel segmentation on high-resolution 3D CT angiography scans." in ISBI, pp. 1280-1283, 2012.

[3] J. Tschirren, G. Mclennan, K. OPalagyi et al., "Matching and anatomical labeling of human airway tree.," IEEE Trans Med Imaging, vol. 24, no. 12, pp. 1540-1547, 2005.

[4] T. Kitasaka, T. Egusa, S. Ota et al., "A method for nomenclature of abdominal arteries extracted from. 3D abdominal CT images based on optimizing combinations of candidate anatomical names," International Journal of Computer Assisted Radiology and Surgery, pp. S45-S49, 2010.

[5] K. Mori, S. Ota, D. Deguchi et al., "Automated Anatomical Labeling of Bronchial Branches Extracted from CT Datasets Based on Machine Learning and Combination Optimization and Its Application to Bronchoscope Guidance," MICCAI 2009, pp. 707-714, 2009.

[6] M. Oda, B. H. Hoang, T. Kitasaka et al., "Automated anatomical labeling method for abdominal arteries extracted from 3D abdominal CT images." in SPIE, pp. 83142F-1-83142F-4, 2012.

[7] W. Zhang, J. Liu, J. Yao et al., "Computer-aided mesenteric small vessel segmentation on high-resolution 3D contrast-enhanced CT angiography scans." in Proc. SPIE, Medical Imaging, pp. 83151T$83151 \mathrm{~T}, 2012$.

[8] J. Yao, D. Sussman, and R. M. Summers, "Fully automated adipose tissue measurement on abdominal CT." in SPIE Medical Imaging, p. 79651Z, 2011.

[9] J. Yao, S. D. O'Connor, and R. M. Summers, "Computer aided detection of lytic bone metastases in the spine using routine CT images." in ISBI, pp. 512-515, 2007.

[10] W. Zhang, J. Liu, J. Yao et al., "Segmenting the Thoracic, Abdominal and Pelvic Musculature on CT Scans Combining Atlas-based Model and Active Contour Model." in Proc. SPIE, Medical Imaging, To be published, 2013

[11] G. Xiong, and L. Xie, "Automated detection of junctions structures and tracking of their trajectories in 4D images." in Information processing in medical imaging proceedings , pp. 486-497, 2011. 